CERN-PH-TH/2010

CBPF-NF-002/10

\title{
One-dimensional structures behind twisted and untwisted superYang-Mills theory
}

\author{
Laurent Baulieu ${ }^{\dagger \ddagger 1}$ and Francesco Toppan*2 \\ ${ }^{\dagger}$ Theoretical Division $C E R N^{3}$ \\ ${ }^{\ddagger}$ LPTHE Université Pierre et Marie Curie ${ }^{4}$ \\ ${ }^{*}$ CBPF, Rio de Janeiro ${ }^{5}$
}

\begin{abstract}
We give a one-dimensional interpretation of the four-dimensional twisted $N=1$ superYangMills theory on a Kähler manifold by performing an appropriate dimensional reduction. We prove the existence of a 6 -generator superalgebra, which does not possess any invariant Lagrangian but contains two different subalgebras that determine the twisted and untwisted formulations of the $N=1$ superYang-Mills theory.
\end{abstract}

\section{Keywords:}

super-Yang-Mills theory, twisted supersymmetry, supersymmetric quantum mechanics.

MSC numbers: 81Q60, 81T13.

\footnotetext{
${ }^{1}$ email address: baulieu@lpthe.jussieu.fr

${ }^{2}$ email address: toppan@cbpf.br

${ }^{3}$ CH-1211 Genève, 23, Switzerland

${ }^{4} 4$ place Jussieu, F-75252 Paris Cedex 05, France

${ }^{5}$ Rua Dr. Xavier Sigaud 150, cep 22290-180, Rio de Janeiro, RJ, Brazil
} 


\section{Introduction.}

In this paper we investigate the relation between untwisted (Poincaré) and twisted supersymmetry from a one-dimensional point of view. We use as an example both the vector and the scalar supermultiplets of the $N=1, D=4$ twisted superYang-Mills theory. They are defined in terms of the $S U(2)$-invariant decompositions, that can be done on a Kähler manifold, of the spinors. The one-dimensional results are basically obtained by a dimensional reduction and an appropriate gauge-fixing, and then reinterpreted from a one-dimensional superalgebra viewpoint. This work is partially motivated by the intriguing question of understanding the process of oxidizing to higher dimensions the rich supersymmetry structure that can be systematically obtained in one dimension. It also sheds new light to the meaning of the twist for supersymmetric theories.

The sets of one-dimensional supersymmetry generators $Q_{I}$ can be presented in a way that their non-vanishing anticommutators are only present on the diagonal,

$$
\begin{gathered}
\left\{Q_{I}, Q_{J}\right\}=\eta_{I J} H \\
{\left[H, Q_{I}\right]=0 .}
\end{gathered}
$$

The classification of the supersymmetric representations for $\eta_{I I}= \pm 1$ has been the subject, following [1], of many publications $[2,3,4,5,6,7]$. It is derived from the classification [8] (see also $[9,10]$ ) of Clifford algebras of (non-degenerate) $(p, q)$ signature. Here we point out that the dimensionally reduced $N=1, D=4$ twisted theory implies that $\eta_{I I}$ can be possibly zero, so that more effort should be done for understanding systems with a general signature $\mathcal{N} \equiv\left[n_{+}, n_{-}, n_{0}\right]$, with $n_{0} \neq 0\left(n_{ \pm}, n_{0}\right.$ denotes the number of terms on the diagonal that are respectively \pm 1 and 0 and, for $n_{-}=n_{0}=0$, we recover the standard supersymmetry algebra of supersymmetric quantum mechanics [11]). With this generalization we hope that one can describe all possible twisted and untwisted supersymmetric theories. The classification

of non-trivial representations of Clifford algebras with extra anticommuting Grassmann-type parameters and their associated superalgebras is not contained in [8] and, respectively, in [1]. In the absence of a general classification we prove, however, the existence of a specific non-trivial realization, as a result of a dimensional reduction of the twisted $N=1, D=4$ supersymmetry.

The gauge symmetry is a non-trivial feature in higher dimensions, where one usually builds equivariant supersymmetry algebra and the balance between commuting and anticommuting fields is achieved modulo gauge symmetries. One can on the other hand introduce new fields, called shadow fields, such that their transformation laws compensate for the appearance of the gauge transformations in the closure of supersymmetry relations [12]. It is however more illuminating, in view of understanding the correspondence with the simplest possible one-dimensional supersymmetric structures, to perform an appropriate gauge-fixing of the Yang-Mills symmetry, which allows one to reach an exact balance between bosons and fermions without introducing 
shadows. This gauge-fixing automatically implies a formulation in lower-dimensions, where one learns new features about the twist operation.

Our aim is thus to understand which phenomena occur when the twisted and untwisted four dimensional Yang-Mills supersymmetry are projected to lower dimensions. We will briefly recall the main features of the twisted $N=1$ Poincaré supersymmetry, which implies using the holomorphic and antiholomorphic decompositions of the spinors on Kähler manifolds. Then we will start the dimensional reduction by setting one of the gauge field components equal to zero, which implies a dimensional reduction to 3 dimensions, if one preserves a 3-generator superalgebra and a dimensional reduction to 1 dimension, if a 4-generator superalgebra is preserved. In one dimension we obtain representations of the $\mathcal{N}_{\text {superPoinc. }} \equiv[4,0,0]$ and $\mathcal{N}_{\text {topol. }} \equiv[1,1,2]$ superalgebras. They are inequivalent, from a standard algebraic point of view. However, in the language of the path integral, both representations are related by a complexification, followed by a linear mapping and a reality condition. If moreover one considers their 3-generator subalgebras $[3,0,0] \subset[4,0,0]$ and $[1,1,1] \subset[1,1,2]$, which are big enough to determine the Lagrangians for both theories, they end up to be different truncations of a 6-generator algebra $[3,3,0]$. The latter algebra itself is too big to admit an invariant Lagrangian. Altogether, the structure that we found in this analysis is rich enough to deserve an exposition. Our results have generalizations for extended supersymmetry. Moreover, the oxidation of the one-dimensional algebra to $2 n$ dimensions by imposing a $S U(n)$ global invariance seems a promising method $[13]$.

\section{The $N=1, D=4$ vector and scalar multiplets and their twisted symmetries.}

The $N=1$ vector multiplet of the $N=1, D=4$ superPoincaré theory contains one gauge field, one Majorana spinor and one auxiliary scalar boson. The scalar multiplet contains two scalars, one Majorana spinor and two auxiliary bosons. Both sets of fields satisfy off-shell and on-shell equilibrium between the number of bosonic and fermionic degrees of freedom. A field multiplet is conveniently denoted by $\left(n_{b}, n_{f}, n_{\text {aux }}\right)$, where $n_{b}, n_{f}$ and $n_{\text {aux }}$ are respectively the numbers of its propagating bosons, propagating fermions and auxiliary boson fields, all defined modulo gauge invariance. The off-shell equilibrium means $n_{f}=n_{b}+n_{\text {aux }}$. The $N=1$ vector and scalar multiplets are thus $(3,4,1)$ and $(2,4,2)$. On a Euclidean 4-manifold with $S U(2)$ holonomy the $N=1$ superYang-Mills theory can be expressed in twisted form, both for the vector and the scalar multiplets $(3,4,1)$ and $(2,4,2)$. This has been studied from various points of view in $[14,15,16,17,18,19,20,21,22]$. To build the twisted formulation one describes the spinors as holomorphic and antiholomorphic forms, in such a way that both multiplets are decomposed 
as follows

$$
\begin{aligned}
& (3,4,1): A_{m}, A_{\bar{m}} ; \psi_{m}, \chi_{\bar{m} \bar{n}}, \chi ; h . \\
& (2,4,2): \Phi, \bar{\Phi} ; \psi_{\bar{m}}, \chi_{m n}, \bar{\chi} ; B_{\bar{m} \bar{n}}, T_{m n} .
\end{aligned}
$$

Here we use the complex space coordinates $z^{m}, z^{\bar{m}}$, where $m=1,2$ are $S U(2)$ indices and $\bar{m}=\overline{1}, \overline{2}$ are the complex conjugate ones. One has both a metric tensor $g_{i j}$ and a complex structure $J_{j}^{i}$, with $J^{2}=-\mathbf{1}, J_{m \bar{n}}=-J_{\bar{n} m}, J_{m n}=0=J_{\bar{m} \bar{n}}=0$. One can express the scalar product in term of $J$ according to the formula $X \cdot Y=g_{i j} X^{i} Y^{j}=i J_{m \bar{n}}\left(X^{m} Y^{\bar{n}}-X^{\bar{n}} Y^{m}\right)$. The raising and lowering of tensorial indices can be expressed in terms of $J$, through the formulas $X^{m}=-i J^{m \bar{n}} X_{\bar{n}}$ and $Y^{\bar{m}}=i J^{\bar{m} n} Y_{n}$.

The link between the Euclidean spinors $\lambda^{\alpha}, \lambda_{\dot{\alpha}}$ and their holomorphic-antiholomorphic decompositions as in Eq. (2) is given by the following formula ${ }^{6}$

$$
\begin{aligned}
\psi_{m} & =\lambda^{\alpha} \sigma_{\mu \alpha \dot{1}} e_{m}^{\mu}, \\
\chi_{\bar{m} \bar{n}} & =\bar{\lambda}_{\dot{\alpha}} \bar{\sigma}_{\mu \nu \dot{2}}^{\dot{\alpha}} e_{\bar{m}}^{\mu} e_{\bar{n}}^{\nu}, \\
\chi & =\delta_{\dot{2}}^{\dot{\alpha}} \bar{\lambda}_{\dot{\alpha}} .
\end{aligned}
$$

In both sides of this equality we have a total counting of 4 real fields.

We may consider the twist formula (3) as a mere change of variables, such that the Dirac Lagrangian satisfies

$$
\bar{\lambda} \gamma^{\mu} D_{\mu} \lambda=\operatorname{Tr}\left(-\chi^{m n} D_{[m} \psi_{n]}+\chi D^{m} \psi_{m}\right) .
$$

The Yang-Mills Lagrangian $\operatorname{Tr}\left(F_{\mu \nu} F^{\mu \nu}\right)$, modulo a boundary term, satisfies

$$
\frac{1}{2} \operatorname{Tr}\left(F_{\mu \nu} F^{\mu \nu}\right) \sim \operatorname{Tr}\left(\frac{1}{2} F^{m n} F_{m n}+\frac{1}{4}\left(F_{m}^{m}\right)^{2}\right) \sim \operatorname{Tr}\left(\frac{1}{2} F_{m n} F^{m n}-h^{2}+h F_{m}^{m}\right),
$$

where $h$ is an auxiliary scalar field which can be eliminated by a Gaussian integration. Eqs. (3) and (4) are only invariant under $U(2)=S U(2) \times U(1) \subset S O(4)$.

Both multiplets in (2) are the so-called twisted expressions of the vector and scalar multiplets of the $N=1, D=4$ superPoincaré symmetry. The conserved ghost or shadow number and the mass dimension of the fields of the twisted multiplets are detailed in the following table

\begin{tabular}{|c|c|c||c|c|c|}
\hline fields & gh.n. & m.d. & fields : & gh.n. & m.d. \\
\hline$A_{m}$ & 0 & 0 & $\Phi$ & 2 & 0 \\
$A_{\bar{m}}$ & 0 & 0 & $\bar{\Phi}$ & -2 & 0 \\
\hline$\psi_{m}$ & 1 & $\frac{1}{2}$ & $\psi_{\bar{m}}$ & 1 & $\frac{1}{2}$ \\
$\chi_{\overline{m n}}$ & -1 & $\frac{1}{2}$ & $\chi_{m n}$ & -1 & $\frac{1}{2}$ \\
$\chi$ & -1 & $\frac{1}{2}$ & $\bar{\chi}$ & -1 & $\frac{1}{2}$ \\
\hline$h$ & 0 & 1 & $T_{m n}$ & 0 & 1 \\
& & & $B_{m n}$ & 0 & 1 \\
\hline
\end{tabular}

\footnotetext{
${ }^{6}$ We define the Euclidean $\sigma$ matrices as $\sigma_{\mu}=\left(i \tau^{c}, \mathbf{1}_{2}\right)$, where $\tau^{c}$, for $c=1,2,3$, are the Pauli matrices.
} 
One defines the four twisted supersymmetry operators $s, s_{\bar{p}}$ and $s_{q r}$ by the following algebra, with the possibility of gauge transformations of the r.h.s.,

$$
\begin{aligned}
\left\{s, s_{\bar{p}}\right\} & =\partial_{\bar{p}}+\delta^{\text {gauge }}\left(A_{\bar{p}}\right), \\
\left\{s_{\bar{p}}, s_{q r}\right\} & =a J_{\bar{p}[q}\left(\partial_{r]}+\delta^{\text {gauge }}\left(A_{r]}\right)\right)
\end{aligned}
$$

(the remaining anticommutators are all vanishing, which means that, in particular, each one of the operators is nilpotent). The above algebra (with $a$ an arbitrarily given dimensionless constant) is the most general one which is compatible with the various charge assignments. Its generators anticommute with $\partial=d z^{a} \partial_{z^{a}}$ and $\bar{\partial}=d \bar{z}^{a} \partial_{\bar{z}^{a}}$. Their charge assignments are

$$
s \equiv\left(\text { gh.n }=1, m . d .=\frac{1}{2}\right), \quad s_{\bar{p}} \equiv\left(\text { gh.n }=-1, \text { m.d. }=\frac{1}{2}\right), \quad s_{q r} \equiv\left(\text { gh.n }=1, \text { m.d. }=\frac{1}{2}\right) .
$$

Without loss of generality we can consider two cases, either $a=0$ or $a=1$. The $a=0$ case is degenerate. It implies not using the Kähler metric. Power counting allows one to compute the field transformations that are compatible with this algebra. They are given by

\begin{tabular}{|c||c|c|c|}
\hline & $s$ & $s_{\bar{p}}$ & $s_{q r}$ \\
\hline \hline$A_{m}$ & $\psi_{m}$ & $a J_{\bar{p} m} \chi$ & 0 \\
$A_{\bar{m}}$ & 0 & $\chi_{\bar{p} \bar{m}}$ & $a J_{\bar{m}[r} \psi_{q]}$ \\
\hline$\psi_{m}$ & 0 & $F_{\bar{p} m}-a J_{\bar{p} m} h$ & 0 \\
$\chi$ & $h$ & 0 & $D_{[q} \psi_{r]}$ \\
$\chi_{\bar{m} \bar{n}}$ & $F_{\bar{m} \bar{n}}$ & 0 & $-a\left(J_{\bar{m}[r} F_{q] \bar{n}}-J_{\bar{n}[r} F_{q] \bar{m}}\right)$ \\
\hline$h$ & 0 & $D_{\bar{p}} \chi$ & $a^{2}\left(J_{\bar{m}[r} J_{q] \bar{n}}-J_{\bar{n}[r} J_{q] \bar{m}}\right) h$ \\
\hline \hline$\Phi$ & 0 & $-\psi_{\bar{p}}$ & $D_{[q} \psi_{r]}$ \\
$\bar{\Phi}$ & $\bar{\chi}$ & 0 & $-a \chi_{q r}$ \\
\hline$\psi_{\bar{m}}$ & $-D_{\bar{m}} \Phi$ & $B_{\bar{p} \bar{m}}$ & $a J_{\bar{m}[q} D_{r]} \Phi$ \\
$\chi_{m n}$ & $T_{m n}$ & $2 J_{\bar{p}[m} D_{n]} \bar{\Phi}$ & 0 \\
$\bar{\chi}$ & 0 & $D_{\bar{p} \bar{\Phi}}$ & $a \chi_{q r}$ \\
\hline$T_{m n}$ & 0 & $-2 J_{\bar{p}[m} D_{n]} \bar{\chi}+D_{\bar{p}} \chi_{m n}-2 J_{\bar{p}[m} \bar{\Phi} \cdot \psi_{n]}$ & 0 \\
$B_{\bar{m} \bar{n} \bar{n}}$ & $2 D_{[\bar{m}} \psi_{\bar{n}]}+\chi_{\bar{m} \bar{n}} \cdot \Phi$ & 0 & $a\left(J_{\bar{m}[q} D_{r]} \psi_{\bar{n}}-J_{\bar{n}[q} D_{r]} \psi_{\bar{m}}\right)$ \\
\hline
\end{tabular}

The non-trivial case is $a=1$. In this case one can associate to $s, s_{\bar{p}}, s_{q r}$ the four operators $Q^{\alpha}, Q_{\dot{\alpha}}$, with a relation as Eq. (3). One then finds that the following supersymmetry algebra for the $Q$ 's,

$$
\left\{Q^{\alpha}, Q_{\dot{\beta}}\right\}=\sigma_{\dot{\beta}}^{\alpha \mu} \partial_{\mu}
$$

modulo gauge transformations, establishes a link between the $N=1, D=4$ superPoincaré algebra and its twisted version. 
The twisted transformation laws of $(3,4,1)$ are independent from those of $(2,4,2)$, while the converse is true only in the abelian limit, the coupling being due to the transformation laws of the auxiliary fields $B_{\bar{m} \bar{n}}$ and $T_{m n}$. One should also note that for $a=0$ the tensor symmetry $s_{q r}$ is completely degenerated for $(2,4,2)$, but not for the $(3,4,1)$ twisted multiplet.

The most general $s$ and $s_{\bar{p}}$ invariant actions of second order in the derivatives of the bosonic fields correspond to both Lagrangians

$$
\begin{aligned}
& \mathcal{L}_{341}=s \operatorname{Tr}\left(\frac{1}{2} \chi^{m n} F_{m n}+\chi\left(-h+F_{m}^{m}\right)\right), \\
& \mathcal{L}_{242}=s \operatorname{Tr}\left(\frac{1}{2} \chi^{\bar{m} \bar{n}} B_{\bar{m} \bar{n}}+\bar{\Phi}\left(D^{\bar{m}} \psi_{\bar{m}}+\Phi \cdot \chi\right)\right) .
\end{aligned}
$$

They are nothing else than the $N=1 D=4$ Lagrangians, as can be verified by computing the $s$-exact terms and using both equations (4) and (5) [22].

One can then check that $s_{q r}$ is also a symmetry of both actions $\mathcal{L}_{341}$ and $\mathcal{L}_{242}$. The $s_{q r}$ symmetry is thus a redundant symmetry, which is already determined from the invariance under the three generators $s$ and $s_{\bar{p}}$. Such redundant symmetries are also present in higher dimension. They often only close when using the equations of motion. On the other hand, in all studied cases the non-redundant symmetries, which uniquely determine the action, close off-shell. For instance, the anticommutation relations of the redundant $s_{q r}$ symmetry in the $S U(4) \subset S O(8)$ decomposition of $N=2, D=8$ with the $(9,16,7)$ multiplet close only on-shell [work in preparation].

The introduction of the fourth generator $s_{q r}$ is however necessary to untwist the 4 generators $s, s_{\bar{p}}, s_{q r}$ into the 4 superPoincaré generators $Q^{\alpha}, Q_{\dot{\alpha}}$. We will actually investigate how the twisted and untwisted formulations are related in one dimension and we will use the existence of the fourth supersymmetry generator.

\section{The gauge $A_{\overline{2}}=0$.}

One may wish to directly understand the balance between bosons and fermions without referring to gauge invariance by reducing the number of degrees of freedom of the gauge field. This implies a breaking of the gauge symmetry, which turns out to imply a dimensional reduction.

We can for instance set $A_{\overline{2}}=0$. Further constraints are required to maintain the supersymmetry algebra. Indeed, the equations

$$
\begin{aligned}
s_{\overline{1}} A_{\overline{2}} & =\chi_{\overline{1} \overline{2}}, \\
s_{q r} A_{\overline{2}} & =a J_{\overline{2} r} \psi_{q}
\end{aligned}
$$

cannot be preserved when imposing $A_{\overline{2}}=0$. It follows that, in order to preserve the consistency of the subalgebra of the 3 generator $s$ and $s_{\bar{p}}$ acting on the vector multiplet $(3,4,1)$, one must 
set to zero all fields derivatives with respect to the variable $z^{\overline{1}}$. One is reduced to a theory in 3 dimensions with 4 bosons, $A_{m}, A_{\overline{2}}$ and $h$, the non-nilpotent part of the algebra being

$$
\begin{aligned}
& \left\{s, s_{\overline{1}}\right\}=\delta_{\text {global }}^{\text {gauge }}\left(A_{\overline{1}}\right), \\
& \left\{s, s_{\overline{2}}\right\}=\partial_{\overline{2}} .
\end{aligned}
$$

In this theory the $S U(2)$ covariance has disappeared.

In order to maintain the full 4-generator algebra (including $s_{q r}$ ) the constraint is more drastic. As indicated by the $s_{q r}$-transformation law of $\chi_{\overline{1} \overline{2}}$, one must set to zero all field derivatives with respect to the variable $z^{m}$, for $m=1,2$, to preserve the equation $s_{q r} A_{\overline{2}}=$ $a J_{\overline{2}} \psi_{q}$. One then recovers a one-dimensional theory with new features that will be detailed in the next Section. The algebra now reads

$$
\begin{aligned}
\left\{s, s_{\overline{1}}\right\} & =\delta_{\text {global }}^{\text {gauge }}\left(A_{\overline{1}}\right), \\
\left\{s, s_{\overline{2}}\right\} & =\partial_{\overline{2}}, \\
\left\{s_{\bar{p}}, s_{q r}\right\} & =a J_{\bar{p}[q}\left(\delta_{\text {global }}^{\text {gauge }}\left(A_{r]}\right)\right) .
\end{aligned}
$$

Modulo global gauge transformations, its only non trivial anticommutator is $\left\{s, s_{2}\right\}$, which expresses the one-dimensional supersymmetry.

\section{Twisted supersymmetry in one-dimension.}

The gauge-fixing $A_{\overline{2}}=0$ implies that the twisted superalgebra with the four generators $s, s_{\bar{p}}$ and $s_{q r}$ is defined in $D=1$ dimension and closes modulo the (remnants of the) gauge transformations. We can therefore analyze the differences between the $D=1$ dimensional reductions of twisted and untwisted supersymmetries, for both the $(3,4,1)$ and $(2,4,2)$ multiplets.

Modulo gauge transformations, the transformations of the dimensionally reduced twisted vector multiplet $(3,4,1)$ are given by

\begin{tabular}{|c|c|c|c|c|}
\hline & $s$ & $s_{\overline{1}}$ & $s_{\overline{2}}$ & $s_{12}$ \\
\hline$A_{1}$ & $\psi_{1}$ & 0 & $-a \chi$ & 0 \\
$A_{2}$ & $\psi_{2}$ & $a \chi$ & 0 & 0 \\
$A_{\overline{1}}$ & 0 & 0 & $-\chi_{\overline{12}}$ & $a \psi_{1}$ \\
$\psi_{1}$ & 0 & 0 & $\dot{A}_{1}+a h$ & 0 \\
$\psi_{2}$ & 0 & $-a h$ & $\dot{A}_{2}$ & 0 \\
$\chi_{\overline{12}}$ & $-\dot{A}_{\overline{1}}$ & 0 & 0 & $a\left(\dot{A}_{1}+a h\right)$ \\
$\chi$ & $h$ & 0 & 0 & 0 \\
$h$ & 0 & 0 & $\dot{\chi}$ & 0 \\
\hline
\end{tabular}


The mixed transformations can be eliminated by field redefinitions that break the shadow number. Such a breaking is admissible because a tensorial index becomes an internal index in $D=1$. We can express the field redefinitions as

$$
\begin{array}{ll}
z_{1}=A_{1}, & \xi_{1}=\psi_{1}-a \chi, \\
z_{2}=A_{\overline{1}}-a A_{2}, & \xi_{2}=\psi_{1}+a \chi, \\
z_{3}=A_{\overline{1}}+a A_{2}, & \xi_{3}=\chi_{\overline{12}}, \\
g=\dot{A}_{1}+2 a h, & \xi_{4}=\chi_{\overline{12}}-a \psi_{2} .
\end{array}
$$

By introducing the basis of four operators

$$
s_{ \pm}=s \pm s_{\overline{2}}, \quad N_{ \pm}=\frac{1}{a}\left(s_{12} \pm s_{\overline{1}}\right),
$$

one obtains a realization of the $\mathcal{N}=[1,1,2]$ generalized supersymmetry. The four operators $s_{ \pm}$ and $N_{ \pm}$are indeed mutually anticommuting and satisfy

$$
s_{ \pm}^{2}= \pm \partial_{t}, N_{ \pm}^{2}=0 \text {. }
$$

No mixed term occurs for the twisted $(2,4,2)$ multiplet. If one defines

$$
\begin{array}{ll}
w_{1}=\bar{\Phi}, & \mu_{1}=\bar{\chi}, \\
w_{2}=\Phi, & \mu_{2}=\psi_{\overline{2}}, \\
d=T_{12}, & \mu_{3}=\bar{\chi}_{12}, \\
f=B_{\overline{12}}, & \mu_{4}=-\psi_{\overline{1}},
\end{array}
$$

one finds that, for the $a=1$ case, the $(3,4,1)$ and $(2,4,2)$ twisted multiplets transform as follows

\begin{tabular}{|c|c|c|c|c|}
\hline & $s_{+}$ & $s_{-}$ & $N_{+}$ & $N_{-}$ \\
\hline$z_{1}$ & $\xi_{1}$ & $\xi_{2}$ & 0 & 0 \\
$z_{2}$ & $-\xi_{3}$ & $\xi_{4}$ & $\xi_{1}$ & $\xi_{2}$ \\
$z_{3}$ & $-\xi_{4}$ & $\xi_{3}$ & $-\xi_{2}$ & $\xi_{1}$ \\
$\xi_{1}$ & $\dot{z}_{1}$ & $-g$ & 0 & 0 \\
$\xi_{2}$ & $g$ & $-\dot{z}_{1}$ & 0 & 0 \\
$\xi_{3}$ & $-\dot{z}_{2}$ & $-\dot{z}_{3}$ & $\dot{z}_{1}$ & $g$ \\
$\xi_{4}$ & $-\dot{z}_{3}$ & $-\dot{z}_{2}$ & $g$ & $\dot{z}_{1}$ \\
$g$ & $\dot{\xi}_{2}$ & $\dot{\xi}_{1}$ & 0 & 0
\end{tabular}$\quad, \quad$\begin{tabular}{|c|c|c|c|c|}
\hline & $s_{+}$ & $s_{-}$ & $N_{+}$ & $N_{-}$ \\
\hline$w_{1}$ & $\mu_{1}$ & $\mu_{1}$ & $-\mu_{3}$ & $-\mu_{3}$ \\
$w_{2}$ & $\mu_{2}$ & $-\mu_{2}$ & $-\mu_{4}$ & $\mu_{4}$ \\
$\mu_{1}$ & $\dot{w}_{1}$ & $-\dot{w}_{1}$ & $d$ & $d$ \\
$\mu_{2}$ & $\dot{w}_{2}$ & $\dot{w}_{2}$ & $f$ & $-f$ \\
$\mu_{3}$ & $d$ & $d$ & 0 & 0 \\
$\mu_{4}$ & $f$ & $-f$ & 0 & 0 \\
$d$ & $\dot{\mu}_{3}$ & $-\dot{\mu}_{3}$ & 0 & 0 \\
$f$ & $\dot{\mu}_{4}$ & $\dot{\mu}_{4}$ & 0 & 0 \\
\hline
\end{tabular}

Let us stress that the disappearance of the mixed terms allows one to present the twisted transformations in a graphical form, in analogy with the untwisted $D=1$ supersymmetric case $[23]$.

It is natural to examine whether the above transformations can be recovered in terms of linear combinations of the $\mathcal{N}=[3,3,0]$ pseudosupersymmetry defined in [1], by computing its 
action on the $(3,4,1)$ and $(2,4,2)$ multiplets, respectively. The $\mathcal{N}=[3,3,0]$ pseudosupersymmetry is made of the 6 operators $Q_{i}, \bar{Q}_{i}, i=1,2,3$, such that $Q_{i}{ }^{2}=\partial_{t}, \bar{Q}_{i}{ }^{2}=-\partial_{t}$, and they all mutually anticommute. The $\mathcal{N}=[3,3,0]$ pseudosupersymmetry transformations can be written as follows

\begin{tabular}{|c|c|c|c|c|c|c||c|}
\hline & $Q_{1}$ & $\bar{Q}_{1}$ & $Q_{2}$ & $\bar{Q}_{2}$ & $Q_{3}$ & $\bar{Q}_{3}$ & $Q_{4}$ \\
\hline$z_{1}$ & $\xi_{1}$ & $\xi_{1}$ & $-\xi_{4}$ & $-\xi_{4}$ & $\xi_{2}$ & $\xi_{2}$ & $\xi_{3}$ \\
$z_{2}$ & $-\xi_{3}$ & $\xi_{3}$ & $-\xi_{2}$ & $\xi_{2}$ & $-\xi_{4}$ & $\xi_{4}$ & $\xi_{1}$ \\
$z_{3}$ & $-\xi_{4}$ & $\xi_{4}$ & $-\xi_{1}$ & $\xi_{1}$ & $\xi_{3}$ & $\xi_{3}$ & $-\xi_{2}$ \\
\hline$\xi_{1}$ & $\dot{z}_{1}$ & $-\dot{z}_{1}$ & $-\dot{z}_{3}$ & $-\dot{z}_{3}$ & $-g$ & $-g$ & $\dot{z}_{2}$ \\
$\xi_{2}$ & $g$ & $g$ & $-\dot{z}_{2}$ & $-\dot{z}_{2}$ & $\dot{z}_{1}$ & $-\dot{z}_{1}$ & $-\dot{z}_{3}$ \\
$\xi_{3}$ & $-\dot{z}_{2}$ & $\dot{z}_{2}$ & $-g$ & $g$ & $\dot{z}_{3}$ & $-\dot{z}_{3}$ & $\dot{z}_{1}$ \\
$\xi_{4}$ & $-\dot{z}_{3}$ & $-\dot{z}_{3}$ & $-\dot{z}_{1}$ & $\dot{z}_{1}$ & $-\dot{z}_{2}$ & $-\dot{z}_{2}$ & $-g$ \\
\hline$g$ & $\dot{\xi}_{2}$ & $-\dot{\xi}_{2}$ & $-\dot{\xi}_{3}$ & $-\dot{\xi}_{3}$ & $-\dot{\xi}_{1}$ & $\dot{\xi}_{1}$ & $-\dot{\xi}_{4}$ \\
\hline
\end{tabular}$\quad, \quad$\begin{tabular}{c|c|c|c|c|c|c||c|}
\hline \\
\hline$w_{1}$ & $-\mu_{3}$ & $-\mu_{3}$ & $\mu_{2}$ & $\mu_{2}$ & $\mu_{1}$ & $\mu_{1}$ & $\mu_{4}$ \\
$w_{2}$ & $\mu_{4}$ & $\mu_{4}$ & $-\mu_{1}$ & $\mu_{1}$ & $\mu_{2}$ & $-\mu_{2}$ & $\mu_{3}$ \\
\hline$\mu_{1}$ & $d$ & $d$ & $-\dot{w}_{2}$ & $-\dot{w}_{2}$ & $\dot{w}_{1}$ & $-\dot{w}_{1}$ & $-f$ \\
$\mu_{2}$ & $-f$ & $-f$ & $\dot{w}_{1}$ & $-\dot{w}_{1}$ & $\dot{w}_{2}$ & $\dot{w}_{2}$ & $-d$ \\
$\mu_{3}$ & $-\dot{w}_{1}$ & $\dot{w}_{1}$ & $-f$ & $-f$ & $d$ & $d$ & $\dot{w}_{2}$ \\
$\mu_{4}$ & $\dot{w}_{2}$ & $-\dot{w}_{2}$ & $d$ & $-d$ & $f$ & $-f$ & $\dot{w}_{1}$ \\
\hline$d$ & $\dot{\mu}_{1}$ & $-\dot{\mu}_{1}$ & $\dot{\mu}_{4}$ & $\dot{\mu}_{4}$ & $\dot{\mu}_{3}$ & $-\dot{\mu}_{3}$ & $-\dot{\mu}_{2}$ \\
$f$ & $-\dot{\mu}_{2}$ & $\dot{\mu}_{2}$ & $-\dot{\mu}_{3}$ & $\dot{\mu}_{3}$ & $\dot{\mu}_{4}$ & $\dot{\mu}_{4}$ & $-\dot{\mu}_{1}$ \\
\hline
\end{tabular}

Here a seventh column has been added to express the action of the fourth generator $Q_{4}$, of the larger $\mathcal{N}=[4,0,0]$ supersymmetry. The action of $Q_{4}$ is in fact fixed, up to an overall sign, once the transformations of the fields under $Q_{1}, Q_{2}$ and $Q_{3}$ have been determined ${ }^{7}$.

For the $(3,4,1)$ twisted multiplet one can identify

$$
s_{+} \equiv Q_{1}, \quad s_{-} \equiv \bar{Q}_{3}, \quad N_{-} \equiv \frac{1}{2}\left(\bar{Q}_{2}-Q_{2}\right) .
$$

Similarly, a 3 -generator subalgebra of the $(2,4,2)$ twisted multiplet can be embedded into the $\mathcal{N}=[3,3,0]$ pseudosupersymmetry through

$$
s_{+} \equiv Q_{3}, \quad s_{-} \equiv \bar{Q}_{3}, \quad N_{-} \equiv \frac{1}{2}\left(Q_{1}+\bar{Q}_{1}\right) .
$$

The operator $N_{+}$, on the other hand, cannot be obtained as a linear combination of the $Q_{i}$ 's and $\bar{Q}_{i}$ 's, for both the $(2,4,2)$ and $(3,4,1)$ twisted mutiplets.

It is worth noticing that, for the $(2,4,2)$ multiplet, the subalgebra generated by $s_{ \pm}, N_{-}$can be recovered as a subalgebra of the $\mathcal{N}=[2,2,0]$ pseudosupersymmetry, since only $Q_{1}, Q_{3}, \bar{Q}_{1}$ and $\bar{Q}_{3}$ enter Eq. (23).

An important remark is that the twisted matter multiplet $(2,4,2)$ cannot be recovered from a dressing $[1,2]$ of the twisted gauge multiplet $(3,4,1)$ (by identifying the extra auxiliary field with either $\dot{z}_{1}, \dot{z}_{2}$ or $\dot{z}_{3}$ ).

To summarize, the 3-generator subalgebra made of $s$ and $s_{\bar{p}}$ induces, after suitable redefinitions of the generators, an $\mathcal{N}=[1,1,1]$ generalized supersymmetry. The 4-generator algebra obtained with the addition of $s_{q r}$ induces in $D=1$ an $\mathcal{N}=[1,1,2]$ generalized supersymmetry. One could wonder whether the representations of the $\mathcal{N}=[1,1,2]$ generalized

\footnotetext{
${ }^{7}$ The dimensional reduction of the untwisted vector and matter multiplets must produce the $(3,4,1)$ and $(2,4,2)$ linear representations of the Euclidean $\mathcal{N}=[4,0,0]$ superalgebra $(1)[24,25]$.
} 
supersymmetry on the twisted multiplets could be recovered from the known $\mathcal{N}=[3,3,0]$ pseudosupersymmetry representations. This is not the case. At most, one can embed a 3-generator subalgebra $\mathcal{N}=[1,1,1] \subset \mathcal{N}=[1,1,2]$ into the $\mathcal{N}=[3,3,0]$ pseudosupersymmetry. This $\mathcal{N}=[1,1,1]$ subalgebra, such that $\mathcal{N}=[1,1,2] \supset \mathcal{N}=[1,1,1] \subset \mathcal{N}=[3,3,0]$, is generated by $s, s_{\overline{2}}$ and a linear combination of $s_{12}$ with $s_{\overline{1}}$. It should be stressed that the inequivalent $\mathcal{N}=[1,1,1] \subset \mathcal{N}=[1,1,2]$ subalgebra generated by $s, s_{\overline{2}}$ and $s_{\overline{1}}$ is not contained in $N=[3,3,0]$. An explicit computation proves that, for both these $N=[1,1,1] \subset N=[1,1,2]$ embeddings, each one of these 3-generator invariances fixes the same Lagrangian, and thus determines the full $N=[1,1,2]$ 4-generator invariance.

As a final remark, let us mention that the twisted and untwisted supersymmetry in $D=1$ can be regarded as acting on the same set of component fields. However, they only admit one common generator.

\section{Conclusions and outlook.}

The algebra of the Poincaré supertranslations is given by 4 generators. In a twisted form, however, a 3-generator subalgebra is sufficient to determine the invariant actions. The consistency of the gauge-fixed 3-generator subalgebra produces a $D=3$ theory. On the other hand, the consistency of the full gauge-fixed 4-generator algebra induces a one-dimensional theory. We have seen that the gauge-fixed one-dimensional reduction of the twisted supersymmetry differs from the dimensional reduction of the untwisted supersymmetry. The latter is a supersymmetry with $\mathcal{N}=[4,0,0]$ supercharges (their squares are positive and coincide with the Hamiltonian), while the former is a $\mathcal{N}=[1,1,2]$ generalized supersymmetry, where one operator has a positive square, one operator has a negative square and the two remaining ones are nilpotent. This has been checked on the vector and the scalar multiplets of the $N=1, D=4$ theory. In this example the untwisted and twisted supersymmetry only share one common generator and are not equivalent, in the usual sense of superalgebra representations, although a complexification, followed by a linear mapping and a reality condition, suggests their very close link, that can be called the twist. We have shown that the twisted $\mathcal{N}=[1,1,2]$ supersymmetry acting on the vector and the matter multiplets cannot be obtained as a result of an embedding into an $\mathcal{N}=[3,3,0]$ pseudosupersymmetry. In contrast, the 3 -generator algebras $\mathcal{N}=[3,0,0]$ and $\mathcal{N}=[1,1,1]$, which are two distinct subalgebras of the $\mathcal{N}=[3,3,0]$ pseudo-supersymmetry, can be obtained. They admit the same invariant Lagrangian, modulo field redefinitions.

Extending the present investigation to the dimensional reduction of the $N=2 D=4$ twisted SuperYang-Mills theory is rather straightforward. It produces a one-dimensional twisted supersymmetry realized on the $(5,8,3)$ set of fields. Much more interesting is the application to the twisted version of the $N=4$ superYang-Mills theory with its $(9,16,7)$ multiplet. The latter theory is conformally-invariant and, in its planar limit, integrable hierarchies are recovered. 
The most relevant part consists of a twisted 6-generator subalgebra which closes off-shell and uniquely determines the theory. The dimensional reduction to low dimensions $(D=1,2)$ allows one to use such powerful tools like the Lax pairs to analyze the integrable properties of the theory. We leave this investigation for future works.

\section{Acknowledgments}

F.T. is grateful to the LPTHE for the hospitality. The work was partially supported by Edital Universal CNPq, Proc. 472903/2008-0.

\section{References}

[1] A. Pashnev and F. Toppan, J. Math. Phys. 42 (2001), 5257 (hep-th/0010135).

[2] Z. Kuznetsova, M. Rojas and F. Toppan, JHEP 0603 (2006), 098 (hep-th/0511274).

[3] C.F. Doran, M. G. Faux, S. J. Gates Jr., T. Hubsch, K. M. Iga and G. D. Landweber, math-ph/0603012.

[4] C.F. Doran, M. G. Faux, S. J. Gates Jr., T. Hubsch, K. M. Iga and G. D. Landweber, hep-th/0611060.

[5] Z. Kuznetsova and F. Toppan, Mod. Phys. Lett. A 23 (2008), 37 (hep-th/0701225).

[6] Z. Kuznetsova and F. Toppan, Int. J. Mod. Phys. A 23 (2008), 3947 (arXiv:0712.3176).

[7] M. Gonzales, S. Khodaee and F. Toppan, arXiv:1006.4678[hep-th].

[8] M.F. Atiyah, R. Bott and A. Shapiro, Topology (Suppl. 1) 3 (1964) 3.

[9] S. Okubo, J. Math. Phys. 32 (1991) 1657; ibid. 32 (1991) 1669.

[10] H. L. Carrion, M. Rojas and F. Toppan, JHEP 0304 (2003) 040 (hep-th/0302113).

[11] E. Witten, Nucl. Phys. B 188 (1981), 513.

[12] L. Baulieu and G. Bossard, Nucl. Phys. B 753 (2006), 273 (hep-th/0603248).

[13] L. Baulieu, A. Losev and N. Nekrasov, Nucl.Phys. B 522 (1998) 82-104 (hep-th/9707174).

[14] A. Johansen, Int. J. Mod. Phys. A 10 (1995) 4325 (hep-th/9403017).

[15] E. Witten, J. Math. Phys. 35 (1994) 5101 (hep-th/9403195).

[16] A. D. Popov, Phys. Lett. B 473 (2000) 65 (hep-th/9909135). 
[17] T. A. Ivanova and A. D. Popov, J. Math. Phys. 41 (2000) 2604 (hep-th/0002120).

[18] C. Hofman and J. S. Park, Nucl. Phys. B 600 (2001) 133 (hep-th/0010103).

[19] J. S. Park, Commun. Math. Phys. 163 (1994) 113 (hep-th/9304060).

[20] J. S. Park, Nucl. Phys. B 423 (1994) 559 (hep-th9305095).

[21] L. Baulieu, A. Tanzini, J. Geom. Phys. 56 (2006) 2379-2401 (hep-th/0412014).

[22] L. Baulieu, G. Bossard, Phys. Lett. B 632 (2005) 138 (hep-th/0507004).

[23] M. Faux and S. J. Gates Jr., Phys. Rev. D 71 (2005), 065002 (hep-th/0408004).

[24] J. Wess and J. Bagger, Supersymmetry and supergravity, $2^{\text {nd }}$ ed., Princeton Univ. Press (1992).

[25] F. Toppan, POS IC2006, 033 (hep-th/0610180). 